\author{
Journal of Chitwan Medical College 2019;9(30):43-46 \\ Available online at: www.jcmc.cmc.edu.np
}

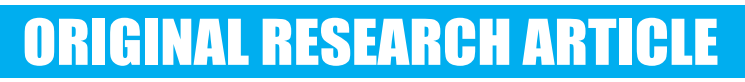

\title{
ULTRASOUND GUIDED PERIPHERAL NERVE BLOCK AS ALTERNATIVE TO SUBARACHNOID BLOCK IN TOTAL HIP REPLACEMENT ARTHROPLASTY: A RETROSPECTIVE STUDY
}

\author{
Anuj Jung Rayamajhi ${ }^{1{ }^{1}}{ }^{*}$, Prashanta Paudel ${ }^{1}$, Subash Chandra Paudel ${ }^{1}$, Bidur Kumar Dhungel ${ }^{1}$, Rupesh Kumar Yadav ${ }^{1}$
}

${ }^{1}$ Civil Service Hospital, Minbhawan, Kathmandu, Nepal

Received: 30 Oct, 2019
Accepted: 19 Dec, 2019
Published: 27 Dec, 2019
Key words: Lumbar plexus block; Sciatic nerve
block; Spinal anesthesia; Total hip replacement;
Ultrasound.
*Correspondence to: Anuj Jung Rayamajhi, Civil Service
Hospital, Minbhawan, Kathmandu, Nepal.
Email: anujrayamajhi@gmail.com

DOI:https://doi.org/10.3126/jcmc.v9i4.26900

Citation

Rayamajhi A, Paudel P, Paudel SC, Dhungel BK, Yadav RK.Ultrasound guided peripheral nerve block as an alternative to subarachnoid block in total hip replacement arthroplasty: a retrospective study.2019;9(30):43-46.



\section{INTRODUCTION}

Continuous development of medical science has increased life expectancy thereby added challenge to medical science to deal with increasing geriatric population. Osteoarthritis of major joint resulting in pain, stiffness, restriction of mobility and atrophy of muscles, and instability and hence disability is common which is often under looked condition in our country. Various non-invasive managements like physiotherapy and oral analgesic are used for analgesia which usually confer short term benefit with significant side effect if used for polling duration. Minimally invasive interventional techniques (intra- articular injections with steroids or viscosupplementation) often fail to provide long-term pain relief. Long term pain relief can be achieved either with Radiofrequency ablation of articular branch of supplying nerves with platelet rich plasma or Total hip replacement. Spinal and epidural anaesthesia are commonly employed for the joint replacement surgeries..$^{1,2}$ However, in geriatric patients there is higher incidence of cardiopulmonary disease with limited cardiopulmonary reserve so spinal and epidural anaesthesia are not well tolerated in all patients leading to potential life threatening events. ${ }^{3}$ With limited mobility assessment of metabolic equivalents (METS) can- not be assed and hence reserve. With the advent of ultrasound guided peripheral nerve minimizing systemic effect can lead to better outcome of the patients. One drawback of multiple peripheral nerve blocks is the need for the large volume of local anaesthetic therefore more chance of its toxicity but this can be easily minimized by using of ultrasound which will aid in the more precise localization of the nerve so less volume of drug can be used. Further continuous catheter can be added to provide postoperative analgesia which enables patients to recover and rehabilitate quickly, reduces medication consumption, and shortens hospital stays. ${ }^{4,5}$ Providing patients with adequate postoperative analgesia might also curtail opioid dependence and abuse, particularly in patients on opioid therapy preoperatively. ${ }^{6}$ The main aim of the surgery is to ambulate the patient as early as possible to regain the normal functional activities of the life thus with the use of peripheral nerve block catheter in the postoperative period will drastically reduce the pain for this purpose.

\section{METHODS}

Retrospective analysis in patients who has undergone lower limb arthroplasty from 2073-3-20 to 2074-4-20 were included 
in study.

All patients were explained regarding procedure in PAC clinic and those who agreed were planned for Peripheral nerve block. In premedication Cap Gabapentin 150-300mg HS and coming morning (CM), tab perinorm $10 \mathrm{mg}$, Inj. Paracetamol $1 \mathrm{gm}$ (IV) and glass of glucose water were given 2 hours prior to surgery.

Patients done in spinal group were designated as Group $S$ and nerve block were as Group P. ASA standard monitoring was done in operating room. Patient was kept in lateral position with limb to be operated above. Inj midazolam $1 \mathrm{mg}$, fentanyl 50 -100 mcg were administered 3 minutes prior as pre procedure sedation. Propofol in dose of $10 \mathrm{mg}$ was added and top up if sedation was not adequate.

Curvilinear 2-6 HZ probe (Mindray M7 ) was used to scan lumbar plexus. Scanning was started from midline in lower lumber region and moved laterally to identify lumbar plexus. Once identified probe adjustment was done to clear transverse process in needle pathway. Stimulating needle (Stimuliplex, B braun) was used. Needle was then introduced in plane techniques and stimulated. Motor response of quadriceps muscle in $0.3 \mathrm{MA}-0.5 \mathrm{MA}$ is considered as adequate response and $1 \%$ Xylocaine with adrenaline of $20 \mathrm{ml}$ was injected. Drug spread was visualized around plexus. Catheterization was done in same setting for post-operative analgesia

Similar probe was used for Sacral plexus. Scanning started from ilium and probe moved downward and medially to identify sacral notch, sciatic nerve and Pudendal muscle. After obtaining motor response of hamstring muscle or dorsiflexion of foot at $0.3-0.5 \mathrm{MA}, 1.5 \%$ Xylocaine with Adrenaline $20 \mathrm{ml}$ was administered.

In same scan $0.3 \%$ Xylocaine with Adrenaline $4 \mathrm{ml}$ was used administered in plane between Gluteus Maximus and pudendal muscle to block Superior Gluteal nerve. Dexmedetomidine infusion at rate of $10 \mathrm{mcg}$ per hour was started and titrated as a part of our standard protocol for all regional anesthesia patient.

Motor and sensory responses were checked after 15 minutes. Pain in dermatome of blocked nerve was considered as failed block. Pain in dermatome cranial to trochanter was considered as missed cutaneous branch of lower thoracic or upper lumbar nerve and local was further added in same area.

Mean arterial blood pressure and all other vitals were regularly measured at interval of 5 minutes throughout the procedure. Intraoperative hypotension and bradycardia were treated with mephentermine $(6 \mathrm{mg})$ and atropine $(0.5 \mathrm{mg})$ respectively. At time of the closure of muscle, the dexmedetomidine infusion was stopped. The patient were transferred to surgical ICU after gaining full consciousness as standard protocol of our institution. For pain management, inj.paracetamol 1gm BD and inj. Ketroloac 30mg BD were given regularly. For rescue analgesics, inj. Fentanyl 25 mcg were given if Numerical rating scale was above 4. Inj. Granisetron $1 \mathrm{mg}$ was used for treating intraoperative nausea and vomiting. During the postoperative period, total fentanyl and incidence of nausea were noted. Intraoperative MAP, fluid consumption, fentanyl consumption, pain scores and incidence of nausea were noted according to protocol. Data were entered in Microsoft excel and analyzed using SPSS software package version 16. Categorical variables were analyzed by using chi square test. Numerical variables were compared by independent $t$ test and $\mathrm{p}$ values were calculated.

\section{RESULTS}

During the study period of the study, 46 patients were selected in which two were excluded from the study as they chose general anesthesia and 1 patient was excluded due to block failure. All operations were successfully completed without any complications. There were no differences in demographics in two groups as shown in Table 1.

\section{Table 1 : Demographic Data}

\begin{tabular}{|l|l|l|l|}
\hline Variables & Group S ( N= 20) & Group $\mathbf{N}(\mathbf{N}=23)$ & p-value \\
\hline Age & $51.90(6.164)$ & $51.13(6.040)^{\times}$ & 0.682 \\
\hline Sex (M/F) & $12 / 8$ & $15 / 8$ & 0.761 \\
\hline
\end{tabular}

Data are expressed in mean $+/-S D$

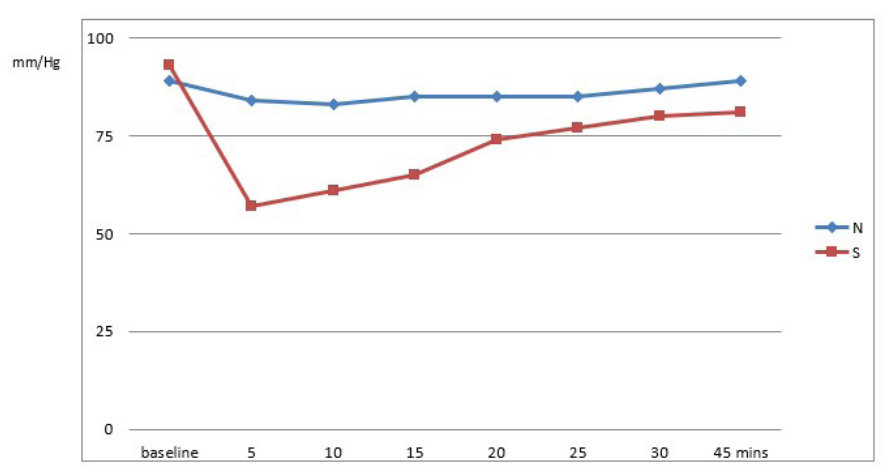

Figure 1: Comparison of MAP between two groups

MAP in Group N vs $S$ at baseline, 5, 10, 15, 20, 25, 30 and 45 minutes respectively : 89.91 vs 93.75 ( $p$-value .113 ), 84.08 vs 57.30 ( $p$-value .000) , 61.95 vs 83.34 ( $p$-value .000$), 85.60$ Vs 65.55 ( $p$-value .000), 85.60 vs 74.65 ( $p$-value .000 ), 85.91 vs 77.05 ( $p$-value .002), 87.26 vs 80.05 ( $p$-value .010), 89.34 vs 81.05 ( $p$-value .036).

As shown in Table 2 there was significant differences in total fentanyl consumption between two study groups as $p$-value .000 .

Table 2: Total Fentanyl Consumption(mcg) between two groups

\begin{tabular}{|l|l|l|}
\hline Group S & Group N & p-value \\
\hline $95.00 \mathrm{mcg}(27.625)$ & $21.739 \mathrm{mcg}(21.721)^{\times}$ & .000 \\
\hline
\end{tabular}

${ }^{\circ}$ Data are expressed in mean $+/-S D$

There were also significant differences in total fluid consumption in two groups ( $p$ value .000) as shown in Table 3. 
Table 3: Total Fluid Consumption between two groups

\begin{tabular}{|l|l|l|}
\hline Group S & Group N & p-value \\
\hline $2060.20 \mathrm{ml}(194.411)$ & $836.30 \mathrm{ml}(129.265)^{*}$ & .000 \\
\hline
\end{tabular}

'Data are expressed in mean $+/-S D$

Table 4: Incidence of Nausea between two groups

\begin{tabular}{|c|c|}
\hline Group S & Group N \\
\hline $5 / 20(20 \%)$ & $1 / 23(4 \%)$ \\
\hline
\end{tabular}

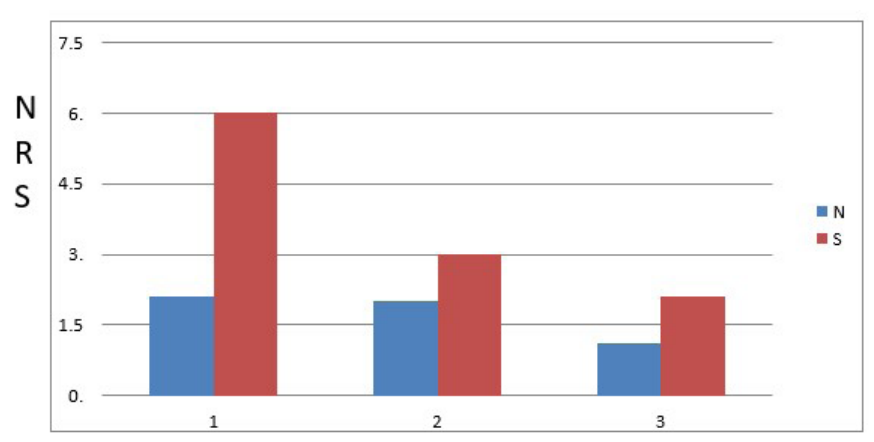

DAY

Figure2: Comparison of NRS between two groups

NRS score between $S$ and $\mathrm{N}$ groups at day 1, 2 and 3 respectively. 6.00 vs 2.17 ( $p$-value .000), 3.00 vs 2.00 ( $p$-value .003), 2.10 vs 1.13 ( $p$-value .000)

\section{DISCUSSION}

Many methods of anesthesia have been employed to perform surgeries ranging from general anesthesia, spinal, epidural anesthesia and peripheral nerve blocks. With the advancement in technology, peripheral nerve block has gained popularity substantially. This outcome can be attributed to the fact that these are elderly patients with other coexisting medical conditions leading to limited cardiac reserve and also their reserve status cannot be properly assessed due to their immobility. Spinal anesthesia can have detrimental effect in this selected group with the increase in morbidity and possibility of unwanted mortality. It is of utmost importance to deliver safe anaesthesia. The use of ultrasonography during peripheral blocks increases their effectiveness and decreases the amount of anesthetic required. ${ }^{7}$ Several studies have evaluated peripheral nerve blocks for lower extremity surgeries ${ }^{8,9}$ and compared them with spinal anesthesia. ${ }^{10}$

The hip joint is innervated by branches of both the LP and the sacral plexus, including the femoral (L2-L4) and obturator nerves ( $L 2-L 4)$, which contain afferents from the anteromedial aspects of the joint, and the sciatic nerve (L4-S3), the nerve to the quadratus femoris (L4-S1) and the superior gluteal nerve (L4-S1), which cover the posterior aspect of the joint. ${ }^{11}$ Complexity of the different variations in innervations of the hip joint has been a great obstacle in providing adequate analgesia and anesthesia for hip arthroplasty by nerve block alone. Few studies have addressed the clinical interest of peripheral nerve or plexus blocks for surgery of the hip. Using a posterior LP technique, Chayen et al. reported successful analgesia in 52 of
57 hip procedures, but recommended combining lumbar and sciatic blocks in this indication and as well for anesthesia. ${ }^{12}$ Although LPB is applied as anesthetic method in a few studies in combination with sciatic block, ${ }^{13}$ its usage is commonly limited to perioperative pain management in hip ${ }^{14}$ and knee surgeries. ${ }^{15}$

Although, difficulties have been documented in terms of using nerve blocks for providing adequate analgesia and analgesia in the past literatures but in our settings, peripheral nerve blocks have become the primary mode of anesthesia for the total hip replacement surgeries.

In this retrospective study, we compared the spinal anesthesia and peripheral nerve blocks technique for THR. As compared to spinal anesthesia, in peripheral nerve block group, there is considerably less amount of fluid administered to the patients prior to the surgical incision $(p=.000)$. This is added benefit of peripheral nerve block as these are geriatric patients who might not tolerate the excess fluid administration. In comparison of hemodynamic parameters (MAP) in both groups, nerve block showed significant hemodynamic stability during the entire procedure as compared to the spinal group which were statistically significant. In a trial that compared general anesthesia alone or combined, either with subarachnoid block or with posterior LP block for femoral neck fracture surgery, White and Chappell ${ }^{16}$ noted greater cardiovascular stability in the group receiving the block which was similar to our study. In spinal anaesthesia group, 10 patients were treated for the hypotension in which majority were managed with the fluid challenge and by giving injection of $6 \mathrm{mg}$ mephenetermine. However, in one of those patient's vasopressor in the form of dobutamine was started and carried out postoperatively for one day to maintain the mean arterial pressure. This can be attributed to spinal induced high block leading to blockage of the cardioaccellerating sympathetic fiber resulting in profound hypotension.

Adequate pain control in the postoperative period correlates to the patient comfort and satisfaction. Lumbar plexus and sciatic nerve catheterization for continuous infusion of local anesthetic for postoperative pain control. NRS score was significantly in nerve block than in spinal group in first operative day. This was similar to the study done by Stevens et $\mathrm{al}^{17}$ described significant lower pain scores at $\mathrm{T}=6 \mathrm{hrs}$ after total hip arthroplasty in patients receiving single shot injection posterior lumbar plexus combined with general anesthesia, compared with the patients who did not receive the block ( VAS 1.4 versus $2.4, \mathrm{P}=.007$ ). They also confirmed that cumulative postoperative morphine consumption at $\mathrm{T}=6$ hours remained significantly lower as well $(5.6 \pm 4.7 \mathrm{mg}$ versus $12.6 \pm 7.5 \mathrm{mg}, \mathrm{P}<.0001$ ) just like fentanyl consumption in our study. A reduction of rescue opioids by the use of a continuous lumbar plexus block also has also been described by Chelly et al. and Siddiqui et al. ${ }^{18}$ Lower pain scores in the post-operative period in nerve block group can be due to inserting catheter for pain management. We did not select to use epidural catheter in spinal group. Use of anticoagulant in arthroplasty surgery from 1st postoperative day add risk of hemodynamic instability 
to devastating complication like epidural hematoma therefore catheterization was avoided. Opioids was used in spinal group; some patients can be very sensitive to the potential danger side effects of opioids but fortunately no respiratory depression or other side effects were noted in the both groups. Another great advantage of good postoperative pain management is that the patients are prepared for early ambulation leading to good surgical outcome.

Incidence of nausea was also higher in spinal group (20\%) than in nerve block (4\%). One patient in spinal group had complained of vomiting which was managed with inj. Metoclopropamide $10 \mathrm{mg}$. Early feeding constitutes towards patient's early recovery. In nerve block group, they were started with feeding within one hour of completion of surgery while in Spinal group, it was much later. As a consequence of early feeding, patients can resume their prescribed medicine within their due schedule so the treatment for their associated illness is not hampered. In five of the patients in the spinal group, patients had complained of acute urinary retention which was treated by one-time urinary cauterization and no such complaints were found in the nerve group. Urinary retention has been well documented complication of spinal anaesthesia and it is further compounded by requiring large amount of fluid in spinal anaesthesia. One patient in nerve block group had to

\section{REFERENCES:}

1. Basques BA, Toy JO, Bohl DD, Golinvaux NS, Grauer JN. General compared with spinal anesthesia for total hip arthroplasty. The Journal of bone and joint surgery. American volume. 2015 Mar 18;97(6):455. [DOI]

2. Mauermann WJ, Shilling AM, Zuo Z. A comparison of neuraxial block versus general anesthesia for elective total hip replacement: a meta-analysis. Anesthesia \& Analgesia. 2006 Oct 1;103(4):1018-25. [DOI]

3. Namba RS, Paxton L, Fithian DC, Stone ML. Obesity and perioperative morbidity in total hip and total knee arthroplasty patients. The Journal of arthroplasty. 2005 Oct 1;20:46-50. [DOI]

4. Jiménez-Almonte JH, Wyles $\mathrm{CC}$, Wyles SP, et al. Is local infiltration analgesia superior to peripheral nerve blockade for pain management after THA: a network meta-analysis. Clin Orthop Relat Res. 2016;474:495-516. [DOI]

5. Andersen KV, Pfeiffer-Jensen M, Haraldsted V, Soballe K. Reduced hospital stay and narcotic consumption, and improved mobilization with local and intraarticular infiltration after hip arthroplasty: a randomized clinical trial of an intraarticular technique versus epidural infusion in 80 patients. Acta Orthop. 2007;78(2):180-6. [DOI]

6. Menendez ME, Ring D, Bateman BT. Preoperative opioid misuse is associated with increased morbidity and mortality after elective orthopaedic surgery. Clin Orthop Relat Res. 2015;473(7):2402-12. [DOI]

7. Koscielniak-Nielsen ZJ, Dahl JB. Ultrasound-guided peripheral nerve blockade of the upper extremity. Curr Opin Anaesthesiol 2012; 25: 2539. [DOI]

8. Sripada R, Reyes JJ, Sun R. Peripheral nerve blocks for intraoperative management in patients with hemophilia A. Journal of clinical anesthesia. 2009 Mar 1;21(2):120-3. [DOI]

9. Touray ST, de Leeuw MA, Zuurmond WW, Perez RS. Psoas compartment block for lower extremity surgery: a meta-analysis. British Journal of Anaesthesia. 2008 Oct 22;101(6):750-60. [DOI] be converted to general anaesthesia with LMA insertion in the intraoperative period due to complaint of pain which may be due to patchy or inadequate block.

Limitations of this study are small size, fixed timed interval for fentanyl consumption and nausea incidence were not applied. Similar post-operative pain management was not applied to both groups as well. Future studies should be a randomized control trial focusing on having large sample size, specified time interval for variable should be used and with same technique should be applied in both groups.

\section{CONCLUSION}

We found that it is possible to conduct hip surgeries under peripheral nerve block to provide adequate analgesia and anesthesia but sound knowledge of ultrasound is pivotal as it gained by experience and expertise to achieve this success. Peripheral nerve blocks can be suitable alternative to spinal, epidural and general anesthesia for hip replacement surgery.

\section{CONFLICT OF INTEREST}

None

\section{FINANCIAL DISCLOSURE}

None

10. Ozhan M, Orhan E, Kurklu M, Demirap B, Suzer A, Cekmen N, Ozhan C, Kurt E. Comparison of peripheral nerve blocks, spinal anesthesia and general anesthesia for ambulatory surgery of the lower limb. Nobel Medicus. 2012 May 1;8(2):73-80. [LINK]

11. Birnbaum K, Prescher A, Hessler S, Heller KD. The sensory innervation of the hip joint-an anatomical study. Surgical and Radiologic Anatomy. 1997 Nov 1;19(6):371-5. [DOI]

12. Chayen $D$, Nathan $H, C$ Chayen $M$. The psoas compartment block. Anesthesiology. 1976;45(1):95-9. [DOI]

13. Ho AM, Karmakar MK. Combined paravertebral lumbar plexus and parasacral sciatic nerve block for reduction of hip fracture in a patient with severe aortic stenosis. Canadian Journal of Anesthesia. 2002 Nov 1;49(9):946-50. [DOI]

14. Chelly JE, Casati A, Al-Samsam T, Coupe K, Criswell A, Tucker J. Continuous lumbar plexus block for acute postoperative pain management after open reduction and internal fixation of acetabular fractures. Journal of orthopaedic trauma. 2003 May 1;17(5):362-7. [DOI]

15. Hadzic A, Karaca PE, Hobeika P, Unis G, Dermksian J, Yufa M, Claudio R, Vloka JD, Santos AC, Thys DM. Peripheral nerve blocks result in superior recovery profile compared with general anesthesia in outpatient knee arthroscopy. Anesthesia \& Analgesia. 2005 Apr 1;100(4):976-81. [DOI]

16. White IW, Chappell WA. Anaesthesia for surgical correction of fractured femoral neck A comparison of three techniques. Anaesthesia. 1980 Nov;35(11):1107-10. [DOI]

17. Stevens RD, Van Gessel E, Flory N, Fournier R, Gamulin Z. Lumbar plexus block reduces pain and blood loss associated with total hip arthroplasty. Anesthesiology: The Journal of the American Society of Anesthesiologists. $2000 \mathrm{Jul}$ 1;93(1):115-21. [DOI]

18. Chelly JE, Casati A, Al-Samsam T, Coupe K, Criswell A, Tucker J. Continuous lumbar plexus block for acute postoperative pain management after open reduction and internal fixation of acetabular fractures. Journal of orthopaedic trauma. 2003 May 1;17(5):362-7. [DOI] 